Cahiers
de a Recherche
sur les Droits Fondamentaux
Cahiers de la recherche sur les droits fondamentaux

17 | 2019

La motivation des actes administratifs. Le droit français à la lumière du droit administratif comparé

\title{
Les fondements de l'obligation de motiver en droit de l'Union européenne : un retour aux sources
}

Jacques Ziller

\section{(2) OpenEdition}

Journals

Édition électronique

URL : https://journals.openedition.org/crdf/3680

DOI : $10.4000 /$ crdf.3680

ISSN : 2264-1246

Éditeur

Presses universitaires de Caen

Édition imprimée

Date de publication : 31 décembre 2019

Pagination : 45-53

ISBN : 978-2-84133-960-0

ISSN : 1634-8842

Référence électronique

Jacques Ziller, «Les fondements de l'obligation de motiver en droit de l'Union européenne : un retour aux sources », Cahiers de la recherche sur les droits fondamentaux [En ligne], 17 | 2019, mis en ligne le 06 février 2021, consulté le 14 novembre 2022. URL : http://journals.openedition.org/crdf/3680 ; DOI : https://doi.org/10.4000/crdf.3680 


\title{
Les fondements de l'obligation de motiver en droit de l'Union européenne: un retour aux sources
}

\author{
Jacques ZILLER \\ Professeur de droit de I'Union européenne à l'université de Pavie (Italie)
}

I. Les sources et fondements de l'obligation de motiver les actes de l'Union européenne

II. Les sources et fondements de l'obligation de motiver les actes des autorités des États membres

III. La différenciation des exigences découlant de l'obligation de motiver selon la nature de l'acte et son destinataire

Le régime de la motivation des actes administratifs en droit de l'Union européenne est particulièrement original pour au moins trois raisons: il date de 1951, époque où il n'y avait pas en Europe d'obligation de motiver les actes en dehors de régimes sectoriels ou limités à un niveau particulier de l'administration; ses fonctions multiples vont bien au-delà de son habituelle fonction corrélée au contrôle juridictionnel; il s'applique avec des modalités différenciées aux institutions, organes et organismes de l'Union elle-même et aux autorités des États membres lorsqu'elles mettent en œuvre le droit de l'Union. Ce régime complexe fait de l'obligation de motiver un pilier central de la garantie de la transparence dans le système de l'Union.

Avec la Charte des droits fondamentaux de l'Union européenne, le droit à une bonne administration a été consacré dans les termes qui suivent:

Article 41 - Droit à une bonne administration

1. Toute personne a le droit de voir ses affaires traitées impartialement, équitablement et dans un délai raisonnable par les institutions, organes et organismes de l'Union.
2. Ce droit comporte notamment:

a) le droit de toute personne d'être entendue avant qu'une mesure individuelle qui l'affecterait défavorablement ne soit prise à son encontre;

b) le droit d'accès de toute personne au dossier qui la concerne, dans le respect des intérêts légitimes de la confidentialité et du secret professionnel et des affaires; décisions.

c) l'obligation pour l'administration de motiver ses

3. Toute personne a droit à la réparation par l'Union des dommages causés par les institutions, ou par ses agents dans l'exercice de leurs fonctions, conformément aux principes généraux communs aux droits des États membres.

4. Toute personne peut s'adresser aux institutions de l'Union dans une des langues des traités et doit recevoir une réponse dans la même langue ${ }^{1}$.

Une lecture hâtive de cette disposition risquerait d'occulter deux caractéristiques importantes de l'obligation de motiver. Il convient d'y ajouter les «Explications »; en effet, selon l'article $6, \$ 1$ du traité sur l'Union européenne (TUE), les dispositions doivent être interprétées

1. Je souligne.

2. Les «Explications» risquent d’être négligées car elles ne sont pas publiées avec la version consolidée des traités, et pourtant leur utilité est indéniable en particulier pour la doctrine; voir J. Ziller, «Le fabuleux destin des Explications relatives à la Charte des droits fondamentaux de l'Union européenne», in Chemins d'Europe. Mélanges en l'honneur de Jean-Paul Jacqué, Paris, Dalloz, 2010, p. 765-781. 
«en prenant dûment en considération les explications ${ }^{2}$ visées dans la Charte, qui indiquent les sources de ces dispositions $»^{3}$. Ce qu'impliquent les explications est que la jurisprudence de la Cour de justice de l'Union européenne (CJUE) relative à l'obligation de motiver est l'une des sources de l'obligation de motiver rappelée par la Charte, que la jurisprudence en question concerne les actes des institutions de l'Union, comme c'est le cas dans l'affaire TU München ou ceux des autorités des États membres comme c'est le cas dans l'affaire Heylens, sur laquelle je reviendrai. Il n'en est pas moins utile de distinguer entre les deux champs d'application de l'obligation en ce qui concerne les fondements de l'obligation, qui en expliquent la portée.

Comme j'ai déjà eu l'occasion de le démontrer par ailleurs ${ }^{4}$, selon le discours dominant sur le principe de transparence en droit de l'Union européenne, c'est l'élargissement vers le nord-est de l'Europe qui aurait conduit à son introduction en droit communautaire. Certes, c'est le traité de Maastricht de 1992 qui a établi - à la demande du Danemark - le principe de la publicité des séances du Conseil ainsi que l'institution du Médiateur européen; et c'est le traité d'Amsterdam de 1997 qui a consacré le droit d'accès aux documents des institutions de l'Union à la demande de la Suède. En réalité, toutefois, l'ancrage dans le droit de l'Union du principe de transparence n'est pas en premier lieu dû aux révisions des traités de 1992 et 1997 sous l'impulsion des pays nordiques, ni d'ailleurs à la jurisprudence de la Cour de justice des Communautés européennes (CJCE), puis de la CJUE, bien antérieure à ces révisions, mais bien au traité fondateur par excellence qu'a été le traité instituant la Communauté européenne du charbon et de l'acier (CECA), source du système institutionnel de l'Union européenne ainsi que de nombre de concepts clés de son droit matériel, en particulier en ce qui concerne le droit du marché intérieur. C'est en effet dès le traité CECA qu'a été établie l'obligation de motiver les actes des institutions de la Communauté en tant que manifestation centrale du principe général de publicité, terme qui était utilisé à l'époque et qui n'a été remplacé que plus tard par celui de transparence. Si l'obligation de motiver a été adoptée dès 1951, c'est pour des raisons propres à la nature de la
CECA, tant du point de vue de son droit matériel, celui de la production et de la commercialisation du charbon et de l'acier, que du point de vue de son droit institutionnel, avec l'établissement d'une Haute Autorité indépendante des gouvernements des États membres. C'est sur cette base que, dès ses premiers arrêts dans les années 1950, la Cour de justice a eu l'occasion de préciser la portée et le contenu de cette obligation. Notons dès à présent que l'obligation du droit de l'Union s'applique à tous les actes, qu'ils soient individuels ou à portée générale et impersonnelle, y compris aux actes législatifs de l'Union, et qu'ils soient de nature juridiquement contraignante ou non.

Le principe fondamental du droit administratif européen selon lequel la mise en œuvre du droit de l'Union est au premier chef une obligation des États membres a eu comme conséquence l'extension d'une obligation pensée d'abord pour les institutions communautaires aux administrations des États membres, avec l'arrêt Heylens de 1987. L'impact de cette jurisprudence a été moins révolutionnaire pour les États membres comme l'Allemagne ou la France, qui à l'époque disposaient déjà d'une législation relative à la motivation des actes administratifs, que pour d'autres, comme par exemple les Pays-Bas, où l'obligation n'existait encore que dans une partie de la législation sectorielle; même pour les pays qui disposaient déjà d'une législation pertinente, l'impact du droit communautaire ne doit pas être sous-estimé, car la motivation en droit communautaire allait plus loin qu'en droit français, comme le démontre justement l'affaire Heylens. Le retour aux sources du droit primaire et de la jurisprudence est nécessaire pour les deux champs d'application du principe de motivation, qu'il reste indispensable de distinguer, car s'il y a des similitudes dans le contenu de l'obligation, il n'y a pas de régime uniforme, ce qui tient en particulier à la différenciation du fondement de l'obligation dans ces deux champs. En effet, les fondements de l'obligation de motivation sont complexes car ils vont bien au-delà de la corrélation habituelle entre motivation et contrôle juridictionnel de l'administration. Les exigences découlant de l'obligation sont différenciées tant selon la nature de l'acte que selon son destinataire, et les conséquences du non-respect de l'obligation de motiver sont variables.

3. Voir Journal officiel de l'Union européenne, C 303, 14 décembre 2007, p. 28, explication de l'article 41: «L'article 41 est fondé sur l'existence de l'Union en tant que communauté de droit dont les caractéristiques ont été développées par la jurisprudence, qui a consacré notamment la bonne administration comme principe général de droit (voir, entre autres, l'arrêt de la Cour du 31 mars 1992, C-255/90 P, Burban, rec. 1992, p. I-2253, ainsi que les arrêts du Tribunal de première instance du 18 septembre 1995, T-167/94, Nölle, Recueil, 1995, p. II-2589; du 9 juillet 1999, T-231/97, New Europe Consulting e.a., Recueil, 1999, p. II-2403). Les expressions de ce droit énoncées dans les deux premiers paragraphes résultent de la jurisprudence (arrêts de la Cour du 15 octobre 1987, aff. 222/86, Heylens, Recueil, 1987, p. 4097, point 15; du 18 octobre 1989, aff. 374/87, Orkem, Recueil, 1989, p. 3283; du 21 novembre 1991, C-269/90, TU München, Recueil, 1991, p. I-5469) ainsi que les arrêts du Tribunal de première instance du 6 décembre 1994, T-450/93, Lisrestal, Recueil, 1994, II-1177; du 18 septembre 1995, T-167/94, Nölle, Recueil, 1995, p. II-2589) et, en ce qui concerne l'obligation de motivation, de l'article 296 du traité sur le fonctionnement de l'Union européenne (voir aussi la base juridique à l'article 298 du traité sur le fonctionnement de l'Union européenne pour l'adoption d'actes législatifs en vue d'une administration européenne ouverte, efficace et indépendante). Le paragraphe 3 reproduit le droit désormais garanti à l'article 340 du traité sur le fonctionnement de l'Union européenne. Le paragraphe 4 reproduit le droit désormais garanti à l'article 20, paragraphe 2, point d), et à l'article 25 du traité sur le fonctionnement de l'Union européenne. Conformément à l'article 52, paragraphe 2, ces droits s'appliquent dans les conditions et limites définies dans les traités. Le droit à un recours effectif, qui constitue un aspect important de cette question, est garanti à l'article 47 de la présente Charte» (j'ajoute les italiques).

4. J. Ziller, "Origines et retombées du principe de transparence en droit de l'Union européenne", in Transparence, démocratie et gouvernance citoyenne, G. J. Guglielmi, É. Zoller (dir.), Paris, Éditions Panthéon-Assas, 2014, p. 25-45. Le présent article reprend un certain nombre des développements de ce chapitre. 


\section{Les sources et fondements de l'obligation de motiver les actes de l'Union européenne}

La disposition fondamentale des traités concernant l'obligation de motiver est l'article 296 alinéa 2 du traité sur le fonctionnement de l'Union européenne (TFUE; ex-article 253 du traité sur le fonctionnement des Communautés européennes 5 , ex-article 199 du traité instituant la Communauté économique européenne - CEE) qui stipule: «Les actes juridiques sont motivés et visent les propositions, initiatives, recommandations, demandes ou avis prévus par les traités». Il s'agit d'une disposition dont l'origine est plus ancienne: l'ancrage dans le droit de l'Union de l'obligation de motiver comme corollaire du principe de publicité ou transparence est l'œuvre du traité fondateur par excellence qu'a été le traité de Paris du 18 avril 1951 instituant la Communauté européenne du charbon et de l'acier, dont les stipulations ont été à juste titre qualifiées de révolutionnaires par Paul Reuter, dans son ouvrage consacré à la CECA, publié à peine un an après l'entrée en vigueur du traité.

Afin d'apprécier pleinement la portée de cette révolution en ce qui concerne la motivation, il faut rappeler qu'au moment de la signature du traité de Paris l'obligation de motiver les décisions d'autorités publiques autres que juridictionnelles n'était établie dans aucun des États fondateurs de la Communauté, ni d'ailleurs dans aucun des autres États actuellement membres de l'Union. La loi de procédure administrative autrichienne de 1925, qui contenait une obligation de motivation des actes administratifs, ne la rendait obligatoire de manière générale que pour les décisions prises en conséquence d'un recours hiérarchique, étant donné les similitudes entre le contrôle par voie de recours hiérarchique et le contrôle par voie de recours juridictionnel dont l'inexistence était ainsi compensée. Seul l'Administrative Procedure Act fédéral de 1946 aux États-Unis avait établi un principe de motivation des décisions administratives, et il était largement ignoré en Europe. La doctrine allemande de droit administratif s'était intéressée à la question dès les années 1920 et avait essayé - sans succès en droit positif - d'établir une théorie générale (Dogmatik) de la motivation, sur la base de textes applicables à quelques matières spécifiques. En France, ce n'est que trois ans après la signature du traité CECA que le Conseil d'État, en l'absence d'une obligation formelle de ce type, allait tirer les conséquences d'un refus de motivation en cours d'instance contentieuse par l'arrêt Barel du 28 mai 1954.
Dans ce contexte, deux dispositions du traité CECA doivent être citées. Selon l'article 15: «Les décisions, recommandations et avis de la Haute Autorité sont motivés et visent les avis obligatoirement recueillis». Le traité CECA a ainsi établi une obligation générale de motivation, qui ne se limite pas aux décisions individuelles ayant un effet négatif pour l'intéressé. Il s'agit d'une disposition largement analysée par la doctrine du droit administratif européen ${ }^{7}$, car elle est reprise par l'article 296, alinéa 2 TFUE. Cette disposition, reprise du traité CECA, qui était déjà présente dans le traité CEE de 1958, avec une modification sur laquelle je reviendrai, a fait l'objet d'une abondante jurisprudence destinée à obliger les autorités concernées à aller au-delà d'un simple visa purement formel, et à préciser les circonstances de fait et les règles de droit qui ont conduit à la décision.

L'article 15 n'est pas la disposition la plus importante du traité CECA en matière de transparence, même si elle en est l'articulation la plus concrète. L'article 5 dudit traité mérite d'être cité intégralement, puisqu'il place l'obligation de motiver dans son contexte:

La Communauté accomplit sa mission, dans les conditions prévues au présent Traité, avec des interventions limitées. À cet effet:

- Elle éclaire et facilite l'action des intéressés en recueillant des informations, en organisant des consultations et en définissant des objectifs généraux;

- Elle met des moyens de financement à la disposition des entreprises pour leurs investissements et participe aux charges de la réadaptation;

- Elle assure l'établissement, le maintien et le respect des conditions normales de concurrence et n'exerce une action directe sur la production et le marché que lorsque les circonstances l'exigent;

- Elle rend public les motifs de son action et prend les mesures nécessaires pour assurer le respect des règles prévues par le présent Traité.

Les institutions de la Communauté exercent ces activités avec un appareil administratif réduit, en coopération étroite avec les intéressés ${ }^{8}$.

L'article 5 du traité CECA est un modèle de rédaction ${ }^{9}$ qui gagnerait à être suivi de nos jours. En quelques mots l'ensemble des aspects de la fonction exécutive de la CECA sont posés, avec les principes qui doivent en guider l'exercice. C'est dans ce contexte que deux termes méritent d'être soulignés - qui figurent tous deux à l'index thématique du livre de Paul Reuter - : les termes motifs et rend public. Dans une section consacrée à «la règle de la publicité», Reuter commente ces dispositions de la manière qui suit:

5. «Les règlements, les directives et les décisions adoptés conjointement par le Parlement européen et le Conseil ainsi que lesdits actes adoptés par le Conseil ou la Commission sont motivés et visent les propositions ou avis obligatoirement recueillis en exécution du présent traité.»

6. P. Reuter, La Communauté européenne du charbon et de l'acier, Paris, LGDJ, 1953.

7. Il suffit à ce propos de se reporter aux manuels de droit institutionnel (ou de droit général) de l'Union européenne, et ce depuis leurs premières éditions, ainsi qu'aux différents commentaires des traités communautaires et de l'Union.

8. Je souligne.

9. Pour comprendre le rôle de Paul Reuter et de Maurice Lagrange dans la conception et la rédaction du traité CECA, il suffit de lire les appréciations que leur dédie Jean Monnet dans ses Mémoires, Paris, Fayard, 1976. 
Ce principe général inspire toutes les règles économiques posées dans le traité: on peut dire sans exagération que selon le Traité le marché commun doit être «une maison de verre». Il suffira de rappeler quelques-uns des exemples essentiels qui seront exposés plus tard: publicité des objectifs généraux et des programmes, et éventuellement des études et informations (article 46) - de toutes données utiles (article 47) - de la liste des avis donnés sur les programmes d'investissements (article 54) - des prix et des conditions de vente des entreprises (article 60) - des décisions relatives aux ententes (article 65), etc. ${ }^{10}$.

Et Reuter de continuer: "La portée de ces dispositions est dans bien des cas absolument révolutionnaire, car la vie économique est basée le plus souvent sur le principe contraire $»^{11}$.

Pour mieux comprendre la pensée de Paul Reuter, et celle des auteurs du traité CECA, et pour replacer la problématique de la publicité / transparence communautaire dans un contexte plus complet, il est à mon avis indispensable de citer également les phrases que Reuter consacre à la concurrence :

La concurrence établie par le Traité n'est pas et ne peut pas être la libre concurrence, mais seulement une concurrence loyale et réglée. La libre concurrence, avalisant toutes les pratiques et s'accommodant de toutes les structures d'entreprises, telle que l'expression juridique en est donnée par le principe classique de l'autonomie de la volonté, a pour premier résultat de fausser la signification économique de la concurrence et de ruiner la concurrence elle-même. Cette appréciation est très familière à certains milieux politiques, mais les systèmes juridiques du continent n'en ont pas, en général, tiré toutes les conséquences, à la différence $d u$ droit des États-Unis. Plus proche de ce dernier, le Traité pose une série de règles qui définissent le contenu de la notion de concurrence loyale et réglée. Cellesci portent non seulement sur la concurrence déloyale au sens habituel, mais aussi sur la validité des pratiques restrictives et des concentrations d'entreprises; la sévérité du Traité, sur ce point, rappelle celle de la législation anti-trust américaine. Mais le Traité va beaucoup plus loin que n'importe quel système aujourd'hui en vigueur, en établissant un double principe de non-discrimination et de publicité, qui s'applique aux dispositions essentielles de toutes les conventions commerciales. Ainsi sont appliquées au commerce du charbon et de l'acier des règles qui, dans un pays comme la France, sont considérées comme définissant le régime très particulier des entreprises dites "services publics». Il n'est pas possible de déterminer encore avec exactitude toutes les conséquences d'une semblable réglementation, mais il est certain qu'elle consacre une révolution économique plus considérable que la plupart des nationalisations effectuées dans les pays continentaux depuis la fin du deuxième conflit mondial ${ }^{12}$.

Ces pages mériteraient d'être plus largement diffusées, afin de contrer les affirmations, courantes dans le débat politique mais aussi dans la doctrine juridique des quinze dernières années, selon lesquelles les traités communautaires seraient l'expression du néo-libéralisme, caché derrière les traits de l'ordo-libéralisme d'Outre-Rhin ${ }^{13}$.

L'on retiendra de ce bref retour sur l'histoire de l'intégration européenne que le concept de maison de verre est inséparable non seulement de la construction institutionnelle des communautés, mais plus encore des fondements du droit matériel progressivement construit depuis plus de soixante-cinq ans, et qu'il a vocation à s'appliquer non seulement aux autorités publiques, mais également au secteur privé - dans le respect des nécessités du secret professionnel, autre concept auquel Reuter consacre d'intéressants développements dans le même ouvrage ${ }^{14}$.

La jurisprudence de la Cour de justice de l'Union, et en particulier les décisions par lesquelles elle dégage des principes généraux du droit, est depuis plus de cinquante ans l'une des sources fondamentales d'importantes déclinaisons du principe de transparence, comme le montre l'exemple de l'arrêt Heylens sur lequel je reviendrai plus loin, auquel s'ajoute une abondante jurisprudence qui a construit peu à peu un droit administratif de l'Union européenne ${ }^{15}$. Rappelons que le premier à avoir proposé d'appliquer la méthode des principes généraux du droit dans le cadre du droit communautaire fut Maurice Lagrange, conseiller d'État et premier avocat général auprès de la Cour de justice de la CECA (bientôt rejoint par l'allemand Karl Roemer). Lagrange - qui avait quelques années auparavant rédigé la première mouture du traité CECA et en particulier les dispositions relatives au contrôle juridictionnel - avait repris dans ses conclusions dans l'affaire Algera $^{16}$ en 1957 la méthode éprouvée du Conseil d'État, s'appuyant sur des sources qui ne sont pas formellement applicables à l'affaire à juger - en l'espèce le droit des États membres, pour une affaire concernant les rapports entre des fonctionnaires communautaires et leur institution de rattachement - pour déduire d'une analyse des droits des États membres un principe général du droit relatif au régime de retrait des décisions individuelles. La méthode avait été reprise par la Cour dans son arrêt du 12 juillet 1957, avant de servir aux rédacteurs du traité de Rome pour mettre au point la disposition qui figure actuellement à l'article 340 TFUE (ex-article 288 TCE), selon laquelle:

10. P. Reuter, La Communauté européenne du charbon et de l'acier, $\$ 74$, p. 76-77.

11. Ibid., $\$ 75$, p. 77

12. Ibid., $\$ 148$, p. $143-144$ (je souligne).

13. Voir, à ce propos, C. Joerges, "Unity in Diversity as Europe's Vocation and Conflicts Law as Europe's Constitutional Form», London School of Economics, LEQS Paper, $\mathrm{n}^{\circ}$ 28/2010, décembre 2010, version révisée: avril 2013.

14. P. Reuter, La Communauté européenne du charbon et de l'acier, $\$ 75-77$, p. 77-79.

15. Voir, en particulier, J. Schwarze, Droit administratif européen, $2^{\mathrm{e}}$ éd., Bruxelles, Bruylant, 2009; Traité de droit administratif européen, $2^{\mathrm{e}}$ éd. J.-B. Auby, J. Dutheil de la Rochère (dir.), Bruxelles, Bruylant, 2014.

16. CJCE, 12 juillet 1957, M $M^{\text {lle }}$ Dineke Algera, M. Giacomo Cicconardi, Mime Simone Couturaud, M. Ignazio Genuardi, Mime Félicie Steichen c. Assemblée Commune de la Communauté européenne du charbon et de l'acier, affaires jointes 7/56, 3/57 à 7/57, ECLI:EU:C:1957:7. 
En matière de responsabilité non contractuelle, l'Union doit réparer, conformément aux principes généraux communs aux droits des États membres, les dommages causés par ses institutions ou par ses agents dans l'exercice de leurs fonctions.

En ce qui concerne plus spécialement l'obligation de motiver, c'est encore Lagrange qui a fourni le premier l'explication la plus articulée de ses fondements, dans ses conclusions dans l'affaire Comptoirs de vente du charbon de la $\mathrm{Ruhr}^{17}$ :

L'obligation pour la Haute Autorité de motiver ses décisions, recommandations et avis, telle qu'elle figure à l'article 15 du traité, répond à une double exigence. Elle constitue d'abord, à l'égard de l'opinion publique, une garantie contre l'arbitraire, en lui permettant de comprendre et de contrôler l'action d'un exécutif investi d'importants pouvoirs. Cela est nécessaire, en particulier, pour l'Assemblée. C'est ce qui explique et justifie que toutes les décisions de la Haute Autorité sont soumises à l'obligation de motivation, même celles qui paraîtraient relever surtout de l'exercice d'un pouvoir discrétionnaire.

Mais l'obligation de motiver est également nécessaire pour permettre le contrôle juridictionnel des décisions pour le cas où elles seraient attaquées devant la Cour. S'agissant d'un contrôle de légalité, exercé normalement par le moyen d'un recours en annulation, qui limite les pouvoirs du juge et implique un partage de compétence, comme de responsabilité, entre ce juge et l'exécutif, selon des frontières souvent délicates à tracer, il est indispensable que la décision fasse clairement apparaître tous les éléments de fait qui doivent permettre au juge de vérifier si elle a été légalement prise ${ }^{18}$.

Il est utile d'ajouter que le caractère supranational de la Haute Autorité est une raison de plus - peut-être d'ailleurs la première dans l'esprit des auteurs du traité pour avoir établi une obligation générale de motiver ses actes: l'indépendance même de la Haute Autorité n'est acceptable pour les gouvernements d'États souverains que si la Haute Autorité est obligée de s'expliquer. C'est d'ailleurs ce que relève la Cour dans son arrêt Allemagne c. Commission du 4 juillet 1963 pour l'article 190 CEE:

[...] en imposant à la Commission l'obligation de motiver ses décisions, l'article 190 ne répond pas seulement à un souci formel, mais vise à donner aux parties la possibilité de défendre leurs droits, à la Cour d'exercer son contrôle et aux États membres, comme à tout ressortissant intéressé, de connaître les conditions dans lesquelles la Commission a fait application du traité ${ }^{19}$.
L'on dirait aujourd'hui que l'obligation de motivation est un outil fondamental de l'accountability des institutions, organes et organismes de l'Union envers la représentation des peuples et des États membres de l'Union. Plus simplement, il est utile de rappeler qu'une fonction essentielle de l'obligation de motiver, en dehors de tout contrôle juridictionnel, est qu'elle doit conduire les autorités publiques à s'interroger sur l'adéquation entre leurs actes et leur mission. C'est d'ailleurs la raison pour laquelle le traité CECA ne limitait pas l'obligation de motiver aux décisions à caractère individuel ou impersonnel et général, mais l'étendait expressément aux avis. Dans l'ensemble, la doctrine s'est relativement peu attachée à la diversité des fonctions de l'obligation de motivation ${ }^{20}$, mais, avec la modification due au traité de Lisbonne qui a remplacé les termes « règlements, directives et décisions » par «actes juridiques», certains commentateurs ont opéré un retour aux sources ${ }^{21}$, et l'attention de la doctrine au fondement institutionnel de l'obligation dans le traité CECA se développe comme il le mérite ${ }^{22}$.

Il serait toutefois erroné de considérer ce fondement de l'obligation de motiver comme le seul dans les traités institutifs et dans le traité CECA. Au contraire, comme le relevait Lagrange dans l'extrait précité de ses conclusions, il s'agissait de faire "clairement apparaître tous les éléments de fait qui doivent permettre au juge de vérifier si [la décision] a été légalement prise». Ce deuxième fondement s'applique également à l'obligation de motiver les actes des États membres dans la mise en œuvre du droit de l'Union.

\section{Les sources et fondements de l'obligation de motiver les actes des autorités des États membres}

Bien que les traités n'évoquent pas la question, et même si la jurisprudence de la Cour de justice limite la portée de l'article 41 de la Charte des droits fondamentaux relatif au droit à la bonne administration aux institutions, organes et organismes de l'Union, ce qui constitue une exception au regard de l'article 51 selon lequel « [1] es dispositions de la présente Charte s'adressent aux institutions, organes et organismes de l'Union dans le respect du principe de subsidiarité, ainsi qu'aux États membres uniquement lorsqu'ils mettent en ouvre le droit de l'Union », il ne fait aucun doute que l'obligation de motiver s'applique également aux autorités des États membres de l'Union car

17. CJCE, 15 juillet 1960, Comptoirs de vente du charbon de la Ruhr, "Präsident», "Geitling», "Mausegatt», et Entreprise I. Nold KG c. Haute Autorité de la Communauté européenne du charbon et de l'acier, affaires jointes 36, 37, 38-59 et 40-59, ECLI:EU:C:1960:36.

18. Conclusions de l'avocat général M. Lagrange sur les affaires jointes 36, 37, 38-59 et 40-59, 24 mai 1960, ECLI:EU:C:1960:27, p. 911 (je souligne).

19. CJCE, 4 juillet 1963, République fédérale d'Allemagne c. Commission de la Communauté économique européenne, affaire 24-62, ECLI:EU:C:1963:14, p. 143 (je souligne).

20. Voir, cependant, C. Hen, «La motivation des actes des institutions communautaires», Cahiers de droit européen, 1977, p. 49 sq.

21. Par exemple M. Geismann, in Europäisches Unionsrecht, H. von der Groeben, J. Schwarze, A. Hatje (dir.), $7^{\mathrm{e}}$ éd., Baden-Baden, Nomos, 2015, vol. IV, p. 1423; voir aussi La codification de la procédure administrative européenne. Le modèle ReNEUAL, J. Ziller, H. Hofmann, J.-P. Schneider (dir.), Bruxelles, Bruylant, 2016, p. 128 sq.

22. Voir, en particulier, J. Mendes, "The Foundations of the Duty to Give Reasons and a Normative Reconstruction ", in The Foundations and Future of Public Law: A Conference in Honour of Paul Craig, L. Fischer, J. King, A. Young (dir.), Oxford, Oxford University Press, à paraître. 
il s'agit d'un principe général du droit de l'Union. Que la source en soit le texte de la Charte, ou la jurisprudence, n'a guère d'importance. Le retour aux sources en l'espèce n'est pas au traité CECA, mais à l'arrêt Heylens, auquel renvoient d'ailleurs les explications pertinentes.

L'arrêt fondamental est celui de la Cour de justice des Communautés du 15 octobre 1987 dans l'affaire Heylens. Cette décision fut rendue sur question préjudicielle du tribunal de grande instance de Lille, qui demandait à la Cour si

Le fait d'exiger, pour exercer les fonctions rémunérées d'entraîneur d'une équipe sportive (article 43 de la loi du 16 juillet 1984), la possession d'un diplôme français ou d'un diplôme étranger admis en équivalence par une commission statuant par avis non motivé et contre lequel aucun recours spécifique n'est prévu constitu[ait] une limitation à la libre circulation des travailleurs définie aux articles 48 à 51 du traite CEE, en l'absence de directive applicable à cette profession $^{23}[$.

Il est utile de remettre également cette décision dans le contexte de l'époque: l'obligation de motiver les décisions négatives avait été introduite en France huit ans avant l'arrêt Heylens par la loi no 79-587 du 11 juillet 1979 relative à la motivation des actes administratifs et à l'amélioration des relations entre l'administration et le public. Elle avait été introduite en Allemagne en 1976, par la loi sur la procédure administrative non contentieuse (Verwaltungsverfahrensgesetz) et dans quelques autres pays européens seulement; et ce n'est qu'à partir de la deuxième partie des années 1980 qu'une telle obligation allait être diffusée dans tous les États membres de l'Union - parfois comme conséquence indirecte de l'arrêt Heylens. L'on remarquera également que la raison d'être de cette obligation de motivation, qui s'applique à toute décision d'une autorité d'un État membre susceptible de porter atteinte à l'exercice d'un droit garanti par les traités communautaires, est la garantie des libertés fondamentales (de circulation des travailleurs, des marchandises, des services et des capitaux) et de l'interdiction des discriminations à raison de l'origine nationale, c'està-dire de principes fondamentaux du marché commun établi par le traité de Rome.

Il est également utile de rappeler les faits, qui sont particulièrement importants en ce qui concerne le droit français. Le plus simple est de citer les conclusions de l'avocat général Giuseppe Federico Mancini:

M. Georges Heylens, ressortissant belge, est titulaire dans son pays d'un diplôme d'entraîneur de football qui lui a été délivré le 18 juin 1977 par l'École des entraîneurs de l'union royale belge des sociétés de football association. Durant la saison sportive 1984-1985, il a été engagé par le Lille Olympic Sporting Club («LOSC»), dont l'équipe joue en première division française. La société a tout de suite tenté de régulariser la situation du nouvel entraîneur, mais, par une lettre du 8 janvier 1985, le ministère de la Jeunesse et des
Sports a informé l'intéressé que la commission nationale des équivalences avait émis un avis négatif sur la reconnaissance de son diplôme comme étant dépourvu d'une valeur égale à celle du titre français correspondant et elle l'a invité à s'abstenir de tout enseignement à titre rémunéré en France.

Toutefois, M. Heylens n'a pas cessé d'entraîner l'équipe de Lille et n'a pas obtempéré à la sommation qui lui a été notifiée ultérieurement par l'Union nationale des entraîneurs et cadres techniques professionnels du football (ci-après «Unectef»). Cette dernière l'a alors cité, ainsi que les responsables du LOSC, devant le tribunal de grande instance de Lille pour qu'ils répondent des délits prévus par l'article 43 de la loi no $84-610$, du 16 juillet 1984 (JORF du 17.7.1984, p. 2288), et par l'article 259 du code pénal relatif à l'usurpation de titres.

Par ordonnance du 4 juillet 1986, le tribunal a sursis à statuer et, en application de l'article 177 du traité CEE, vous a adressé la question suivante:

«Le fait d'exiger, pour exercer les fonctions rémunérées d'entraîneur d'une équipe sportive (article 43 de la loi du 16 juillet 1984), la possession d'un diplôme français ou d'un diplôme étranger admis en équivalence par une commission statuant par avis non motivé et contre lequel aucun recours spécifique n'est prévu constitue-t-il une limitation à la libre circulation des travailleurs définie aux articles 48 à 51 du traité CEE, en l'absence de directive applicable à cette profession?»

[...]

Il est opportun de signaler tout d'abord que, par des lettres du 13 juin et du 19 août 1985, le ministre de la Jeunesse et des Sports a informé M. Heylens qu'il avait reconnu l'équivalence de son diplôme à la suite d'un nouvel examen effectué par la commission spéciale. Le gouvernement français, à qui nous devons l'information, a cependant admis que, opérant ex nunc, cette reconnaissance n'a pas d'incidence sur l'existence éventuelle du délit pénal. Le problème soulevé par les juges de Lille - à qui il appartient en tout cas d'apprécier si l'interprétation demandée à la Cour conserve un intérêt pour leur décision - reste donc pleinement actuel.

Pour fournir à ces juges une réponse vraiment utile, il est toutefois indispensable de modifier dans une certaine mesure la question qu'ils nous posent. En effet, conformément à la législation française en vigueur, il n'est pas exact d'affirmer que c'est la «commission des équivalences » qui décide des demandes de reconnaissance des diplômes étrangers. L'article 6, précité, de l'arrêté susmentionné prévoit, au contraire, que les décisions relatives à ces demandes sont prises, après l'examen effectué par la commission, «par le secrétaire d'État à la Jeunesse et aux Sports». L'avis de la commission apparaissant comme un simple acte préparatoire de la mesure définitive est donc dépourvu d'effets externes et comme tel ne peut pas avoir une incidence sur la situation des intéressés.

S'il en est ainsi et s'il est vrai que le demandeur a le droit d'utiliser, contre l'acte du ministre, les voies de recours normales prévues par l'ordre juridique français, le fait que l'avis non obligatoire de la commission ne soit pas susceptible de recours n'est certainement pas contraire 
au droit communautaire. En mettant l'accent sur ce détail, le tribunal de Lille nous pose donc un problème inexistant; et cela oblige à élargir la portée de sa demande en la formulant dans les termes que nous avons employés au début. Elle doit donc être entendue comme visant à établir si la réglementation communautaire en matière de circulation des personnes exige le respect de certaines conditions minimales, parmi lesquelles une motivation explicite, dans les décisions nationales qui concernent les droits attribués aux migrants ${ }^{24}$.

Cette citation me paraît indispensable pour bien comprendre que la Cour a saisi l'occasion du renvoi préjudiciel du tribunal de Lille pour souligner la portée générale des deux obligations que le droit communautaire établit à la charge des États membres, à savoir l'obligation d'établir des voies de recours aptes à soumettre à un contrôle juridictionnel les décisions susceptibles d'avoir un impact sur l'exercice des libertés garanties par le traité - ce qui est souligné depuis le traité de Lisbonne par l'article 19, $\$ 1$, deuxième alinéa du TFUE, selon lequel «Les États membres établissent les voies de recours nécessaires pour assurer une protection juridictionnelle effective dans les domaines couverts par le droit de l'Union» -, et l'obligation de motiver ces décisions. Le lien entre motivation et contrôle juridictionnel, auquel Lagrange se référait déjà dans ses conclusions dans l'affaire Comptoirs de vente $d u$ charbon de la Ruhr est donc rappelé par l'arrêt Heylens. Comme dans le cas précédent, il faut souligner qu'il s'agit de permettre la réalisation des objectifs du traité, à travers - notamment - la possibilité de recours au juge.

Au point 15 de son arrêt, la Cour établit que:

L'efficacité du contrôle juridictionnel, qui doit pouvoir porter sur la légalité des motifs de la décision attaquée, implique, de manière générale, que le juge saisi puisse exiger de l'autorité compétente la communication de ces motifs. Mais, s'agissant plus spécialement, comme en l'espèce, d'assurer la protection effective d'un droit fondamental conféré par le Traité aux travailleurs de la Communauté, il convient également que ces derniers puissent défendre ce droit dans les meilleures conditions possibles et se voient reconnaître la faculté de décider, en pleine connaissance de cause, s'il est utile pour eux de saisir la juridiction. Il en résulte qu'en pareille hypothèse l'autorité nationale compétente a l'obligation de leur faire connaître les motifs sur lesquels son refus est fondé soit dans la décision elle-même, soit dans une communication ultérieure faite sur leur demande ${ }^{25}$.

Cette décision est particulièrement significative puisque le juge communautaire détache en partie l'obligation de motivation des décisions administratives - lorsqu'elles sont pertinentes pour l'exercice des droits conférés par les traités communautaires - de la procédure juridictionnelle destinée à la protection de ces droits et qu'il le relie à «la faculté de décider» des particuliers. Qui plus est, le contexte de l'arrêt Heylens est particulièrement utile parce qu'il rappelle la problématique essentielle des actes détachables que l'on retrouve aussi bien en droit de l'Union qu'en droit national dans tous les cas où une décision finale dépend d'un avis rendu en amont par une autre autorité que celle qui décide en fin de compte. Il est particulièrement utile de prendre en compte le développement considérable des procédures composites impliquant à la fois les autorités des États membres et les institutions, organes et organismes de l'Union ${ }^{26}$.

\section{La différenciation des exigences découlant de l'obligation de motiver selon la nature de l'acte et son destinataire}

Pour donner suite à l'introduction par le traité de Lisbonne d'une nouvelle base juridique - l'article 298 TFUE - qu'il considère comme idoine pour une codification des principes de la procédure administrative non contentieuse en droit de l'Union européenne, le Parlement européen a adopté le 15 janvier 2013 une résolution par laquelle il demandait à la Commission «de soumettre, sur la base de l'article 298 du traité sur le fonctionnement de l'Union européenne, une proposition de règlement sur un droit européen de la procédure administrative, suivant les recommandations détaillées figurant en annexe», annexe «contenant des recommandations à la Commission sur un droit de la procédure administrative de l'Union européenne ${ }^{27}$. Devant l'immobilité de la Commission, le Parlement européen a par la suite adopté le 9 juin 2016 une deuxième résolution ${ }^{28}$ accompagnée d'une proposition entièrement rédigée de «Règlement du Parlement européen et du Conseil pour une administration européenne ouverte, efficace et indépendante» annexée à cette résolution. Cette proposition de règlement contient un article 19 relatif au «Devoir de motivation» selon lequel:

1. Les actes administratifs énoncent clairement les motifs sur lesquels ils s'appuient.

2. Les actes administratifs indiquent leur base juridique, les faits pertinents et la manière dont les différents intérêts en présence ont été pris en compte.

24. Conclusions de l'avocat général G. F. Mancini sur l'affaire 222/86, 18 juin 1987, ECLI:EU:C:1987:304, p. 4106-4108.

25. CJCE, 15 octobre 1987, Union nationale des entraîneurs..., point 15, p. 4117 (je souligne).

26. Pour plus de développements, voir J. Ziller, «Les concepts d'administration directe, d'administration indirecte et de co-administration et les fondements du droit administratif européen ", in Traité de droit administratifeuropéen, J.-B. Auby, J. Dutheil de la Rochère (dir.), $3^{\mathrm{e}}$ éd., Bruxelles, Bruylant, en préparation.

27. Résolution du Parlement européen du 15 janvier 2013 contenant des recommandations à la Commission sur un droit de la procédure administrative de l'Union européenne (2012/2024(INL)), en ligne: http://www.europarl.europa.eu/sides/getDoc.do?pubRef=-//EP//TEXT+TA+P7-TA-2013$0004+0+\mathrm{DOC}+\mathrm{XML}+\mathrm{Vo} / / \mathrm{FR}$.

28. Résolution du Parlement européen du 9 juin 2016 pour une administration de l'Union européenne ouverte, efficace et indépendante (2016/2610(RSP)), en ligne: http://www.europarl.europa.eu/sides/getDoc.do?pubRef=-//EP//TEXT+TA+P8-TA-2016-0279+o+DOC+XML+Vo//FR 
3. Les actes administratifs contiennent un exposé individuel des motifs pertinents au regard de la situation des parties. Si cela n'est pas possible en raison du grand nombre de personnes concernées, un exposé général des motifs est suffisant. Dans ce cas, toutefois, toute partie qui demande expressément un exposé individuel des motifs doit l'obtenir.

Il s'agit d'une utile tentative de synthèse de la jurisprudence abondante et complexe de la Cour de justice en matière de motivation. Une synthèse récente de sa propre jurisprudence par la Cour est donnée par exemple dans l'arrêt Swedish Match :

[...] selon une jurisprudence constante, la motivation exigée à l'article 296, deuxième alinéa, TFUE doit être adaptée à la nature de l'acte en cause et doit faire apparaître de façon claire et non équivoque le raisonnement de l'institution, auteur de l'acte, de manière à permettre aux intéressés de connaître les justifications de la mesure prise et à la juridiction compétente d'exercer son contrôle. Il n'est pas exigé que la motivation spécifie tous les éléments de fait et de droit pertinents, dans la mesure où la question de savoir si la motivation d'un acte satisfait aux exigences de l'article 296, deuxième alinéa, TFUE doit être appréciée au regard non seulement de son libellé, mais aussi de son contexte ainsi que de l'ensemble des règles juridiques régissant la matière concernée [...].

Il ressort également d'une jurisprudence constante que la portée de l'obligation de motivation dépend de la nature de l'acte en cause et que, s'agissant d'actes à portée générale, la motivation peut se borner à indiquer, d'une part, la situation d'ensemble qui a conduit à son adoption et, d'autre part, les objectifs généraux qu'il se propose d'atteindre. Dans ce contexte, la Cour a précisé, notamment, qu'il serait excessif d'exiger une motivation spécifique pour les différents choix techniques opérés si l'acte contesté fait ressortir l'essentiel de l'objectif poursuivi par l'institution $[\ldots]^{29}$.

Cette synthèse est utile parce qu'elle souligne les deux caractéristiques principales de la jurisprudence.

En premier lieu, la portée de l'obligation de motiver n'est pas la même pour les actes individuels et pour les actes de portée générale. Ce qu'il faut toutefois souligner c'est justement, qu'en droit de l'Union, l'obligation de l'article 296 vaut pour tous les actes juridiques, qu'ils aient une portée générale ou soient des décisions individuelles, et, dans ce dernier cas, qu'il s'agisse d'une décision à portée négative pour le destinataire ou non.

Qui plus est, il n'y a aucune distinction selon l'auteur de l'acte. Bien au contraire, depuis que le traité de Maastricht a ajouté aux règlements, directives et décisions adoptés par le Conseil ou la Commission, ceux «adoptés conjointement par le Parlement européen et le Conseil », il est évident que l'obligation de motiver s'applique aussi bien aux actes à caractère législatif qu'aux autres, ce qui va bien au-delà de l'obligation de motiver des droits nationaux, qui se limite en général aux actes administratifs.
La formulation de l'article 296 qui résulte du traité de Lisbonne ajoute deux nouveautés. La première est de mettre en évidence le fait que l'obligation de motiver ne lie pas seulement la Commission, le Conseil et le Parlement européen, mais bien toutes les institutions (y compris donc le Conseil européen et la Banque centrale européenne en particulier) ainsi que les organes (Comité économique et social et Comité des régions) et organismes de l'Union. C'est la conséquence du fait que le traité de Lisbonne a consacré la solution jurisprudentielle selon laquelle le contrôle juridictionnel est ouvert contre les actes de ces organismes, en particulier des agences de l'Union.

La seconde nouveauté tient au fait que, à l'instar du traité CECA, le TFUE impose l'obligation de motiver non seulement pour les actes à portée obligatoire, mais également pour les avis, recommandations et autres actes de l'Union. Il n'est pas inutile de souligner que, à l'exception des mesures de gestion et de cas exceptionnels comme par exemple l'Office de l'Union européenne pour la propriété intellectuelle, les agences n'adoptent pas en général des actes juridiques obligatoires, mais essentiellement des avis et recommandations, auxquels s'applique donc bien évidement l'obligation de motiver. Une limite importante résulte du fait que le contrôle juridictionnel ne permet pas de mettre en cause la validité d'un acte non contraignant; mais l'absence de motivation d'actes non contraignants qui doivent être considérés comme des actes préparatoires.

Il résulte de tout ce qui précède qu'en cas de recours en annulation ou de renvoi préjudiciel en validité fondé sur un non-respect de l'obligation de motiver, la jurisprudence ne peut qu'être variée dans les exigences qu'elle impose aux institutions, organes et organismes de l'Union. L'on pourra toujours discuter de l'adéquation du degré de sévérité de ces exigences, qui varie non seulement selon la nature de l'acte - contraignant ou indicatif -, mais aussi selon son auteur - le législateur, la Commission ou un organisme - ou l'auteur du recours dans le cas d'un recours en annulation - requérant privilégié, destinataire d'une décision, ou personne qu'un acte regarde directement et / ou individuellement - ou encore organe juridictionnel d'un État membre dans le cadre d'un renvoi préjudiciel. Pour les mêmes raisons, les conséquences que la Cour tire d'une motivation insuffisante en termes d'éventuelle annulation varient en fonction non seulement de l'auteur de l'acte, de sa nature et du requérant, notamment parce qu'un acte sans portée obligatoire ne peut pas faire l'objet d'un recours en annulation, mais que l'annulation subséquente sur la base de la motivation insuffisante d'un acte préparatoire dépend des circonstances et en particulier de la possibilité ou non d'appliquer une théorie des actes détachables. Tenter de synthétiser en quelques lignes cette jurisprudence, qui ne peut qu'évoluer selon les affaires qui seront soumises à la Cour, est donc à mon avis une gageure. 
Quant à la question de savoir quelles sont les exigences découlant de l'obligation de motiver et les conséquences d'une motivation inexistante ou inadéquate des actes des autorités des États membres, il me paraît aventureux d'essayer d'établir une théorie générale sur la base de la jurisprudence. Les réponses sont fortement conditionnées par les réponses que le droit de la procédure administrative, contentieuse et non contentieuse ${ }^{30}$ apporte dans le cadre de chaque État membre, si bien qu'étendre à un État membre une solution établie par la Cour pour une question préjudicielle en provenance d'un autre État membre risque fort de conduire à des contresens. C'est la principale raison de fond qui m'a conduit à limiter cet exposé pour l'essentiel à ce qui fait l'originalité de l'obligation de motiver en droit de l'Union: la multiplicité de ses fondements. 